\title{
ON GROUPS WITH SLOW INTERSECTION GROWTH
}

\author{
MARTIN KASSABOV AND FRANCESCO MATUCCI
}

\begin{abstract}
Intersection growth concerns the asymptotic behavior of the index of the intersection of all subgroups of a group that have index at most $n$. In this note we show that the intersection growth of some groups may not be a nicely behaved function by showing the following seemingly contradictory results: (a) for any group $G$ the intersection growth function $i_{G}(n)$ is super linear infinitely often; and (b) for any increasing function $f$ there exists a group $G$ such that $i_{G}$ below $f$ infinitely often.
\end{abstract}

\section{INTRODUCTION}

Let $\mathcal{G}$ be a class of subgroups of a group $\Gamma$. We define the $\mathcal{G}$-intersection growth function of $\Gamma$ by letting $i_{\Gamma}^{\mathcal{G}}(n)$ be the index of the intersection of all $\mathcal{G}$-subgroups of $\Gamma$ with index at most $n$. In symbols,

$$
i_{\Gamma}^{\mathcal{G}}(n):=\left[\Gamma: \Lambda_{\Gamma}^{\mathcal{G}}(n)\right], \quad \text { where } \Lambda_{\Gamma}^{\mathcal{G}}(n):=\bigcap_{[\Gamma: \Delta] \leq n, \Delta \in \mathcal{G}} \Delta .
$$

Here, $\mathcal{G}$ will always be either the class of all subgroups, the class $\triangleleft$ of normal subgroups or the class max $\triangleleft$ of maximal normal subgroups of $\Gamma$, i.e. those subgroups that are maximal among normal subgroups. The corresponding intersection growth functions will then be written $i_{\Gamma}(n), i_{\Gamma}^{\triangleleft}(n)$ and $i_{\Gamma}^{\max \triangleleft}(n)$.

Intersection growth has been first defined by Biringer, Bou-Rabee and the authors in [1] where it has been studied for free groups and some arithmetic groups and a connection has been drawn between intersection growth and the residual finiteness growth, which will be mentioned at the end of this introduction.

The aim of this note is to build examples of groups whose intersection growth behaves slowly at certain integers and much faster at others. We will assume all groups in this note to be finitely generated. For a group $\Gamma$, let $R(\Gamma)$ denote the intersection of all finite index subgroups of $\Gamma$. Obviously, $i_{\Gamma}^{\mathcal{G}}(n)=i_{\Gamma / R(\Gamma)}^{\mathcal{G}}(n)$, so we can assume that $R(\Gamma)=\{1\}$, i.e. we will assume $\Gamma$ to be finitely generated and residually finite.

Observation 1. For any infinite residually finite group $\Gamma$ one has that $i_{\Gamma}(n) \geq$ $n$ for infinitely many positive integers $n$. 
The main result of this note states that, in some sense, the opposite is also true.

Theorem 2. For any strictly increasing function $f: \mathbb{N} \rightarrow \mathbb{N}$ there exists a finitely generated residually finite group $\Gamma$ such that $i_{\Gamma}(n)<f(n)$ for infinitely many positive integers $n$.

Remark 3. We observe that Observation 1 and Theorem 2 are still true if we replace $i_{\Gamma}(n)$ with either $i_{\Gamma}^{\triangleleft}(n)$ or $i_{\Gamma}^{\max } \triangleleft(n)$. The proofs are easy adaptations of the ones we give below and we omit them to keep this note short.

Observation 1 and Theorem 2 show that intersection growth functions may behave in a bad way. More precisely, these functions cannot be approximated via regular functions (polynomial, exponential, etc.) as they have different behavior at different integers.

The main idea is to construct a group with a very limited number of finite quotients and this is achieved by taking a suitable direct product of a family of simple groups.

Recall that for a residually finite finitely generated group $\Gamma=\langle X\rangle$, one can define the residual finiteness growth function $F_{\Gamma, X}(n)$ as the minimal natural number $N$ such that any element of word length $\leq n$ with respect to $X$ can be detected in a quotient $Q$ of cardinality $\leq N$ (see Bou-Rabee [3] for an introduction). All the constructions in this paper can immediately be translated to get similar information about residual finiteness growth. See Bou-Rabee and Seward [2] for a similar result about building examples with arbitrarily large residual finiteness growth functions. We also mention the work of Kharlampovich, Myasnikov and Sapir [6] who build finitely presented solvable examples with arbitrarily large residual finiteness growth functions.

Finally, we recall that for a group $\Gamma$, one defines $r_{\Gamma}(n)$ to be the number of inequivalent finite irreducible $n$-dimensional representations of $G$ (assuming this number is finite). One defines $R_{\Gamma}(n)=\sum_{k=1}^{n} r_{\Gamma}(k)$ to be the representation growth function of $\Gamma$. Craven [4] showed analogues of Observation 1 and Theorem 2 for $R_{\Gamma}(n)$. Unlike Observation11 Craven's upper bound Theorem $\mathrm{G}$ requires a more careful and involved construction.

\section{PROOFS OF THE RESULTS}

Proof of Observation 1 . Since $\Gamma$ is infinite and residually finite for every $n$ there exists a finite index subgroup $H_{N}<\Gamma$ of index $\left[\Gamma: H_{N}\right]=M>n$. This clearly implies that $\Lambda_{\Gamma}(N) \leq H_{N}$, i.e., $i_{\Gamma}(N) \geq N$. 
Observation 4. Let $\left\{n_{i}\right\}$ be a strictly increasing sequence of positive integers, such that $n_{1} \geq 5$. If $\mathfrak{G}:=\prod_{i=1}^{\infty} \operatorname{Alt}\left(n_{i}\right)$, then

$$
i_{\mathfrak{G}}\left(n_{k}-1\right)=\prod_{i<k}\left|\operatorname{Alt}\left(n_{k}\right)\right| .
$$

Proof. The group $\mathfrak{G}$ is profinite and endowed with the product topology. Notice that for profinite groups is natural to define intersection growth with respect to finite index closed subgroups. In this case by Nikolov and Segal [8] every subgroup of finite index is closed, but we do need to use this fact. We observe the following:

(1) if $H \leq \mathfrak{G}$ has index $\leq n_{k}-1$, then $H \cap \operatorname{Alt}\left(n_{k}\right)$ has index $\leq n_{k}-1$ inside $\operatorname{Alt}\left(n_{k}\right)$,

(2) $\operatorname{Alt}\left(n_{k}\right)$ has no nontrivial subgroups of index $\leq n_{k}-1$, but it has subgroup of index $n_{k}$.

Therefore, if $H \leq \mathfrak{G}$ is a closed subgroup of index $\leq n_{k}-1$, then $H \cap$ $\operatorname{Alt}\left(n_{i}\right)=\operatorname{Alt}\left(n_{i}\right)$, for any $i \geq k$. This implies that

$$
H \geq \overline{\bigoplus_{i \geq k} \operatorname{Alt}\left(n_{i}\right)}=\prod_{i \geq k} \operatorname{Alt}\left(n_{i}\right)
$$

and therefore $\Lambda_{\mathfrak{G}}\left(n_{k}-1\right) \geq \prod_{i \geq k} \operatorname{Alt}\left(n_{i}\right)$. It is very easy to see the opposite inclusion (since the group $\prod_{i \geq k} \operatorname{Alt}\left(n_{i}\right)$ can be realized as the intersection of point stabilizers of the actions of $\mathfrak{G}$ via $\operatorname{Alt}\left(n_{i}\right)$ on $n_{i}$ points for $i \leq k$ ), which concludes the proof.

This observation shows that if the sequence $\left\{n_{k}\right\}$ grows very quickly then, in some sense, the function $i_{\mathfrak{G}}$ is very small at certain values.

Remark 5. The group $\mathfrak{G}$ constructed in Observation 4 is not finitely generated, but it is finitely generated as a profinite group.

The idea is to construct a finitely generated group whose profinite completion is the same as the group $\mathfrak{G}$ constructed in Observation 4 . Since $\mathfrak{G}$ is finitely generated as a profinite group it is very easy to find finitely generated dense subgroup $\Gamma \subseteq \mathfrak{G}$. Such an embedding will give a surjective map $\pi: \widehat{\Gamma} \rightarrow \mathfrak{G}$, however this map is often not injective because $\Gamma$ might have finite index subgroups which which are not visible in $\mathfrak{G}$ [9, 7] and can not be used to obtain information about $i_{\Gamma}$. If the map $\pi$ is an isomorphism it is very easy to see that $i_{\Gamma}=i_{\mathfrak{G}}$ (see [1]).

For some time it was not known if it is possible to find $\Gamma$ such that the map $\pi$ is an isomorphism. This question was settled in Theorem 1.2 in [5], which we restate here in the weaker form we need.

Theorem 6 (Kassabov-Nikolov, [5]). For any strictly increasing sequence $\left\{n_{k}\right\}$, the Cartesian product $\prod_{k=1}^{\infty} \operatorname{Alt}\left(n_{k}\right)$ is a profinite completion of a finitely generated residually finite group. 
We are now ready to prove Theorem 2 .

Proof of Theorem 2 We choose $n_{1}=5$ and, inductively, we define $n_{k}$ in the following way: given $n_{k}$, let $n_{k+1}$ be an integer large enough such that $\prod_{i \leq k}\left|\operatorname{Alt}\left(n_{k}\right)\right|<\min \left\{n_{k}+1, f\left(n_{k+1}-1\right)\right\}$.

The chosen sequence allows us to construct the group $\mathfrak{G}$ of Observation 4 .

By Theorem 6 we have that $\mathfrak{G}=\widehat{\Gamma}$, for a finitely generated residually finite group $\Gamma$. As was observed above $i_{\Gamma}=i_{\mathfrak{G}}$ and so $i_{\Gamma}\left(n_{k}-1\right)=\prod_{i<k}\left|\operatorname{Alt}\left(n_{k}\right)\right|$. By construction $i_{\Gamma}\left(n_{k}-1\right)<f\left(n_{k}-1\right)$ for any $k>1$ and we are done.

\section{ACKNOWLEDGEMENTS}

The first author was partially supported by NSF grants DMS-0900932 and DMS-1303117. The second author gratefully acknowledges the Fondation Mathématique Jacques Hadamard (FMJH - ANR - Investissement d'Avenir) for the support received during the development of this work. We are grateful to Khalid Bou-Rabee for helpful mathematical conversations.

\section{REFERENCES}

[1] I. Biringer, K. Bou-Rabee, M. Kassabov, and F. Matucci. Intersection growth in groups. submitted. arXiv:math. GR/0812221.

[2] K. Bou-Rabee and B. Seward. Arbitrarily large residual finiteness growth. submitted, arXiv:math.GR/1304.1782.

[3] Khalid Bou-Rabee. Quantifying residual finiteness. J. Algebra, 323(3):729-737, 2010.

[4] David A. Craven. Lower bounds for representation growth. J. Group Theory, 13(6):873-890, 2010.

[5] Martin Kassabov and Nikolay Nikolov. Cartesian products as profinite completions. Int. Math. Res. Not., pages Art. ID 72947, 17, 2006.

[6] O. Kharlampovich, A. Myasnikov, and M. Sapir. Algorithmically complex residually finite groups. submitted, arXiv:math.GR/1204.6506.

[7] B. H. Neumann. Groups Whose Elements Have Bounded Orders. J. London Math. Soc., S1-12(2):195-198.

[8] Nikolay Nikolov and Dan Segal. On finitely generated profinite groups. I. Strong completeness and uniform bounds. Ann. of Math. (2), 165(1):171-238, 2007.

[9] László Pyber. Old groups can learn new tricks. In Groups, combinatorics \& geometry (Durham, 2001), pages 243-255. World Sci. Publ., River Edge, NJ, 2003.

Martin Kassabov

Department of Mathematics, Cornell University

Malott Hall, Ithaca, NY 14850, USA

E-mail: kassabov@math.cornell.edu

Francesco Matucci

Département de Mathématiques, Faculté des Sciences d'Orsay, Université Paris-Sud 11, Bâtiment 425, Orsay, France

E-mail: francesco.matucci@ math.u-psud.fr 